\title{
Cultural Life: Theory and Empirical Testing
}

\author{
Authors: Dan C. Baciu ${ }^{1,2 *}$.
}

Affiliations: ${ }^{1}$ University of California Santa Barbara Department of English, Santa Barbara, CA 93106, USA. ${ }^{2}$ University of California Santa Barbara Department of the History of Art and Architecture, Santa Barbara, CA 93106, USA.

*Corresponding author. Email: books@yahoo.com ORCID: 0000-0002-0043-5616

ABSTRACT: "What is life?" and Erwin Schrödinger's answer, "negative entropy", inspired researchers in the 20th century to unite physics, chemistry, and physiology into a new synthesis that many believe to be an important foundation for life science today. Decades after Schrödinger, life scientists are still fascinated by the riddle that entropy can only accumulate in physical systems, which often leads to biological deterioration, but life finds ways to persist and prevail. So to say, life "negates" entropy through other, equally physical processes. Can this fascination and research concept be broadened even further to human culture? Short after Schrödinger's publication, Claude Shannon coined the term "information entropy." Information entropy accumulates when noise interferes during communication. Yet, from this observation, something surprising can be inferred. Cultural life and biological life have something in common: Both cultural and biological life persists and prevails in spite of entropic processes. Does this insight mean that cultural life also negates entropy in Schrödinger's sense? These questions guided me over several years of research, during which I developed and tested a new theory of culture based on variation-selection processes and homeostatic regulation. My contribution is to discover that these two processes not only explain how biological systems negate entropy. They also explain some of the most important phenomena of cultural life: waves of fashion, polarization, diversification, cycles of growth and reform, and the formation of common ethos across entire bodies of knowledge. With access to big data and supercomputing, I tested my theory against hundreds of thousands of news, magazines, books, and TV transcripts, as well as textual content collected from the social media. Historical, institutional, and geographical information was extracted from these data using a new method; and new interactive tools were created to interpret the results. What should not be missed when reading this article is that the theory proposed here reveals a striking equivalence between nature and culture. The article states this equivalence in mathematical terms, and it traces its roots back to the most relevant context in the history of science. The mathematical breakthrough is relevant because it aligns the humanities to science while also allowing for live evaluation of what I call "cultural diversification cycles."

Keywords: Variation-selection processes, homeostatic regulation, cultural life, entropy, negative entropy, constructal law, Shannon communication, quasispecies equation, LotkaVolterra equations, diversification processes, Science, Chicago School.

Link to article: https://osf.io/ad9fu/ 


\section{Introduction}

Over the last few decades, causal explanation models from the life sciences have entered linguistics and helped advance the field (1-4). My present article expands on earlier work by shifting the focus even further from language to culture. Culture is the integration of all human thought, which, of course, is frequently expressed through language.

Despite the slight shift of focus, the main thread of my article remains a question that is fundamental to biology: The systems under consideration have the ability to persist and prevail, but how do they achieve this?

Biology is often faced with this question because all biological systems are under pressure to persist and prevail in spite of entropic processes. Homeostasis is the biological response to this latter kind of physical degradation and death. If you wish, the question of how organisms persist and prevail is a question of life and death. Biology, as the Greek name indicates, is the science of life. Homeostasis is an important condition for life. My article suggests that homeostasis also applies to cultural life.

The question, what life is, fascinates not only biologists. Homeostasis was already a well defined concept when the quantum physicist Erwin Schrödinger popularized the idea of negative entropy (5). Shortly thereafter, the mathematician Alfred Lotka formulated a third law of thermodynamics stating that evolution negates entropy (6). Lotka's formulation was replaced decades later by the constructal law (7), which states the conditions under which flow systems persist and prevail. Homeostasis, negative entropy, and constructal law could seem almost selfevident and only of practical utility, had they not united biology with physics (8), and would they not yield a potential new answer the role of the humanities among these sciences.

In this present article, I propose that entire groups of authors and audiences establish variation-selection processes and homeostasis, which, in turn, helps them cope with entropic processes. This theory is formulated in words as well as mathematics, and it is tested against empirical data.

The mathematics itself is so well known though its applications in biology that it requires little separate attention in this article and is placed in the supplement wherever possible. This allows me to focus on discussing theory, testing results, and historical roots.

Next to the figures printed with this article there also are interactive interfaces available online. The laboratory under my direction at UC Santa Barbara has helped develop workflows and design interfaces to facilitate my research (9). Please forgive that some of the visuals are more beautifully designed than usual. Designers and art students have had their hand in designing, and we feel that the eyes can more than read letters.

What may surprise the theoretical biologist at the end of the day is that my theory makes predictions and explains a broad array of cultural phenomena without breaking out of standard evolutionary reasoning. The clear ties to pre-existent knowledge and mathematics will make it increasingly easy to evaluate and understand culture live, outside laboratories, and they also make me ask: does the study of cultural life still need to stand apart from life science? 


\section{Large-scale dissemination: variation-selection processes negate entropy}

Information entropy was coined as an analog to what physicists call entropy principle. In physics, entropic processes transform all useful energy into heat, in any closed system. The system eventually reaches physical dead state. During communication, a similar process occurs. When messages are transmitted from senders to receivers, they accumulate noise and information entropy. This is to say, all useful information disappears behind noise. This law is not only supported by observation, but, if communication is defined as exact or approximate reproduction of a message at a new place, the law can also be logically deduced from the definition. Claude Shannon chose this framework in 1948 (10).

Broadening this definition to large-scale dissemination leads us to a surprising, new proposition. When many messages are exactly or approximately reproduced at a new place, entropy no longer accumulates within the system. The reason is that, under the new circumstances, communication accommodates large-scaled variation-selection processes. The mathematical structure that results from this new definition is equivalent to the quasispecies equation, known in physical chemistry to play an important role in the evolution of life (11). Large-scale communication, as defined here, and quasispecies evolution are mathematically equivalent (12).

To fully appreciate the meaning of this mathematical equivalence, one must recognize that not only noise but also human thinking and creativity can be defined as approximate reproduction. In human culture, variants are created through creative processes: Scientists redesign their methods; designers think in variants and alternatives; humanists rethink their evaluation criteria; and even memories are rewritten when they are recalled, which occasionally changes them (13). Rethinking, redesigning, and rewriting are three slightly different actions, but they have the same effect. They create variants. Let us call this phenomenon variation principle.

Here again, one must predict that entropy accumulates during isolated acts of cultural reproduction. Variation introduces changes, but useful solutions are rare, and they are therefore likely to be lost. Anthropologists have described this phenomenon from first-hand observation. In small populations, knowhow is often degraded when it is passed on. People make changes to the techniques that they learn, and those changes more often prove to degrade the initial performance of the techniques in question (14).

The opposite must be predicted when multiple creative acts occur in parallel, on a sufficiently large scale. At any time, new audiences have the choice between multiple variants that are being disseminated next to each other. There are original, improved, plagiarized, and defective variants, all of which exist in parallel. The audience has the choice.

This process is called a variation-selection process, and it can be modeled with the quasispecies equation. Brainstorming is a similar process because variants are being created and evaluated in parallel. Would then variation-selection processes be the initial cause for diversification in human culture? Are there any limits? How far can variation-selection processes take us?

\subsection{Variation across nature and culture, and its limits}

"Varieties may indefinitely depart from the original type," this is the observation that inspired Alfred Russel Wallace to formulate the theory of evolution on his hunt of paradise birds in Indonesia. He meant biological varieties, but it might have dawned to him that the same 
observation can also be made among varieties of thought. Thought variants can bring us anywhere, as well. One can combine and recombine any set of ideas. Wallace's later travelogue dwells on the varieties of names that people and commerce had given to paradise birds: god's birds, dead birds, etc. There exist many variant names for the same species $(15,16)$.

Yet, are there limits to variation? Wallace together with Charles Darwin repeatedly revisited the terms varieties and species. Their articles, jointly read at that now famous meeting of the Linnaean society in 1858, as well as their earlier and later work, substantiated that the distinction between varieties and species is only one of degree (15-19). Surprisingly, this tenet brought Linnaean taxonomy only closer to its Aristotelian roots and to the study of human culture. Long before Linnaeus, Aristotle and his school used genus and differentia to define any idea or develop any taxonomy (20). This practice surfaced again in evolutionary thinking, and it remains today. In line with the historical roots, there are today also literary genres and chemical species. Aristotelian logic served as the basis of systematic thinking in all fields of study, from biology to the humanities and to chemistry.

The quasispecies equation was developed when it became possible to chemically analyze genes. This historical alignment made it possible, with respect to chemical species, to refine and test evolutionary theory and also better understand the limits of variation (21). Both biologists and chemists use systematic thinking in their work, but the chemists' perspective enriched the study of evolution because some processes are better visible in chemistry. There, the theoretical framework is clearly defined: One gene is a chemical species. A group of variant genes is a quasispecies.

One main insight from the quasispecies equation must be discussed here: When multiple variants are being reproduced in parallel, the result is a distribution in which some variants are more frequent than others. This distribution of frequent versus rare variants depends on both, how useful each variant is, and how strong variation is.

In absence of variation, only one variant survives, namely the one that is being reproduced at the fastest rate. Variation instantly broadens this distribution. In presence of variation, the unit of selection is no longer the isolated variant, but the quasispecies of variants. While the fastest reproducing variants predominate, some other variants are being reproduced along with them, simply because they are related. Each quasispecies is comprised of a set of useful genes together with their less prolific but related variants (21).

Our proposition, again, is that large-scale dissemination in human culture can be modeled in an equivalent system. Quasi-species means literally approximately reproduced species. Approximate reproduction is not limited to genetics. As already mentioned, Shannon spoke of approximate reproduction in human communication. Ultimately, approximate reproduction has the same effect in both nature and culture. Regardless whether it occurs through genetic mutation, recombination, or through human creativity, the effect of approximate reproduction is that it creates variants. The logical consequence is a system in which reproduction, variation, and unequal reproductive rates lead to large-scale accumulation - not of entropy, but of increasingly meaningful variants.

In our case, a quasispecies is a group of variant ideas. Variation interconnects these variants. Together, they are a unit of evolutionary selection. The rate of growth of each variant within one group is equal to the collective rate of growth of the entire group. Thus, if the group grows, all variants grow. In addition, if a new variant grows faster due to new circumstances in 
the environment, the entire quasispecies will be pulled in that direction. These properties help quasispecies more efficiently explore the limitless possibilities that culture provides.

While quasispecies consist each of multiple variants, they are nevertheless limited in size. No quasispecies will produce all possible variant ideas at any given time; each quasispecies will only produce a number of similar, variant ideas. This sets limitations to the directions in which variation alone can search. There always remain a variant not tested, a stone not turned, and a road not taken.

These theoretical insights raises two questions: 1) Can we confirm the existence of groups of ideas as units of evolutionary selection that can be analyzed with the quasispecies equation? 2) If so, will we find only one unit, or do many of them coexist?

If multiple quasispecies coexist, we must consider whether next to variation-selection processes, there also is an additional process of self-regulation. Unaided by other processes, multiple quasispecies can coexist only in the unusual limit of exactly equal growth rates. Biologists easily recognize the problem. Cancer grows only slightly faster than regular tissue, but it grows large enough to kill unless it is checked. (If the rate of growth of $a$ is greater than that of $b$, then it is always much greater.) In healthy organisms, growth rates are controlled by homeostatic regulation. This means that the coexistence of multiple quasispecies can be interpreted as a hint that homeostatic regulation controls the interplay between them. Before we get to this point, let us first work with the quasispecies concept.

\subsection{Variation-selection processes explain power laws and clustering patterns}

The existence of quasispecies of ideas is easily confirmed, and it explains the two most visible empirical laws of human culture: power laws and clustering patterns. These empirical phenomena cannot be neglected. Countless researchers have studied them. Even school kids know of power laws. Teachers call them pyramids of income, or pyramids of fame. Clustering patterns are even more visible. Everyone experiences clustering on an every-day basis. Clustering is the root of almost all cultural groups and categories. Of course, we know that categories are always blurry. And this, too, is at once explained by the quasispecies equation. The blur consists of those aforementioned variants that go along because of variation. In addition, the quasispecies equation explains why power laws and clusters persist and prevail. Variation-selection processes, as captured by the quasispecies equation, are a basic mechanism that makes these phenomena self-perpetuating. Empirical testing is shown in Figures 1-3 and is discussed below in brief and in detail in the supplement.

Let us discuss power laws and clusters in the order in which they were discovered. Vilfredo Pareto, Alfred Lotka, and George Zipf were among the first to observe that money, culture, and language live in power laws (22-24).

Towards the end of the 19th century, Pareto evaluated income and found that few winners take most. When he observed and quantified this distribution, he compared it to a pyramid. Pareto's imagery immediately entered popular culture, for example through the title sequence of Fritz Lang's silent movie "Metropolis." The sequence features a pyramid of buildings in a city with high income disparity.

Around the time of Metropolis, Lotka went another, more scientific approach. He counted author names in bibliographies. He discovered the same distribution. Few authors get most citations. The mathematics led to the development of bibliometrics. A similar idea also entered 
linguistics: Few words are frequent; many are rare. Power laws in natural language were eventually dubbed Zipf's laws, after Zipf popularized them at Harvard.

However, evaluating power laws and drawing Pareto plots has an Achilles heel. It requires a researcher to first choose a variable, for example income, and then sort the data by that very same variable. This latter step makes each Pareto plot look like a straight falling line on logarithmic paper (hence the image of a pyramid. The slope of the Pareto plot is imagined as the slope of the pyramid.) However, the sorting procedure also is the reason why Pareto plots are considered statistically incomplete (25). The quasispecies equation replicates the Pareto plots in a statistically complete setup while also explaining the mechanism behind the Pareto distribution in a broader evolutionary context (Fig.1 and Supplement).

Fig.1 One quasispecies. The Chicago schools of sociology and related fields are studied as a quasispecies of ideas. Records from public media are structured into four subgroups. The prevalence of each subgroup is estimated using the quasispecies equation. The results are within the expected boundaries. Stochastic refinements from Bertels et al. 2017.

A Quasispecies simulation scheme. B Quasispecies evolution over time (left). Quasispecies distributions (right). C Distributions from empirical data on Chicago schools of sociology and related fields. (Data: Chicaog school corpus. $y=1 \mathrm{~K}$ references.)

\section{Wild type 2. Variants sourrounding 1. 3. Variants surrounding 2.4. All other variants}
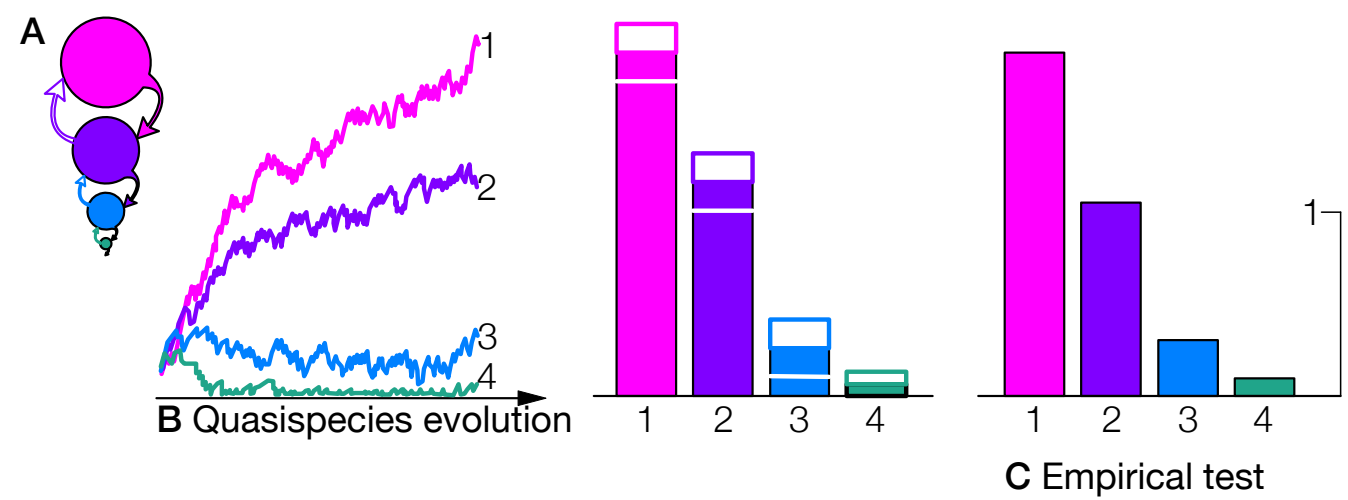

Power laws were first quantified much before clustering. Income data, journal impact, word frequencies, or the like can be drawn as straight-lined Pareto plots, but in many instances, the same journals can also be grouped into themes that remain hidden on the previous visualization. Theoreticians who attempted to explain the Pareto distribution overlooked this result (25). Those fateful clusters were only later observed, mostly through computer-driven techniques of dimensionality reduction.

A social scientist, Pierre Giraud, may have been one of the first and few who searched for an explanation that united power laws and clusters. He reduced natural language to a handful of units he called semes and believed to be universal (26). Later authors spoke of memes, while dimensionality reductions independently advanced the development of entire fields of study such 
as digital sociology and digital humanities. Digital somewhat reflects that the physical meaning of those computer-driven techniques remained hidden.

Around the turn of the millennium, Thomas Landauer and Susan Dumais combined a new modeling algorithm with an empirical test to assess model quality, which paved the way towards increasingly better-ranking technical solutions of dimensionality reduction (27-29). In most of these approaches, collections of written text were processed into so-called word co-occurrence matrices. The matrices were then further processed trough a procedure called $t f$-idf, followed by eigendecomposition, or by variants and generalizations thereof. The precise role of these steps was disputed while researchers attempted various ex-post rationalizations. They puzzled: what did the technical solution really solve?

The path that I followed in my research comes the opposite direction and gives a coherent answer. Variation-selection processes led me to the quasispecies equation. To solve the equation, one must first estimate the rates of exact reproduction and variation, followed by eigendecomposition. Mathematically, the procedure developed over the empirical path is equivalent. One way to estimate variation rates is by collecting text, saving it into matrices, and performing tf-idf. To simulate the flow of information through the system on must then proceed with eigendecomposition. A step-by-step description is given in the supplement.

The quasispecies equation was the missing piece of the puzzle between Shannon communication and clustering patterns. The next section intuitively explains important properties of the quasispecies and tests additional predictions.

\subsection{Groups of ideas speciate through variation-selection processes}

Eigendecompositions, in our setting, simulate large-scale dissemination. Students familiar with matrix operations have an easy time understanding the details. They imagine each matrix as a multidimensional space. They also know of eigenvectors, picturing them as the independent axes of the matrix. In our case, each quasispecies is an eigenvector. It is an independent axis of the matrix.

Take your own hands as an illustrative example. You have ten fingers but only two hands. Within the framework of your body, the fingers on one hand are not independent axes, they often move together. Press your small finger and you'll see the fourth finger move as well. Stretch your arm in one direction, and all fingers go along. In contrast to the fingers, the hands are independent axes. You can stretch them out independently. In this analogy, each finger is an idea, each hand is a quasispecies.

In the case of cultural life, we are studying dissemination. Hence, the eigenvectors can be interpreted as independent axes of dissemination. Along each axis, information is exactly or approximately reproduced at an overall distinct pace. Technically speaking, each eigenvector has an eigenvalue, which represents the rate at which the axis grows. You can multiply an eigenvector by the matrix, but you really get the same result if you multiply the eigenvector by its growth rate, namely the eigenvalue. This property makes eigenvectors self-perpetuating; which explains why clustering patterns persist and prevail. No matter how the system grows, the variants along one axis grow together. They move together like fingers on one hand. The eigenvalue is the speed with which you stretch out your hand.

Mathematically, the largest quasispecies must come to predominate. Take the illustrative analogy of the hand again: Although your hands are the same length, one of them, left or right, 
likely is your dominant hand that you choose to use most. However, empirical results on human culture show that, in human culture, multiple quasispecies coexist. Culture has many hands. Before continuing to the interpretation of this important result, let us use the quasispecies framework to make and test one more prediction.

If the dissemination axes, as proposed here, result from variation-selection processes, this also means that they are realms of speciation, potentially shaped by geography, history, and institutions. This proposition can be empirically confirmed (Fig.2, 3, and Supplement).

Geography is not only important in cultural life but also in the study of biological life. Puzzling geographical distributions of fauna and flora brought Wallace and Darwin's theory of evolution into existence. Indonesia (15) and Galapagos (19) offered first sources of insight. Later, island biogeography transformed ecology from descriptive field into theoretical and mathematical science (30). Biological speciation very often is defined by the geographical space.

The new findings on cultural life suggest that variation-selection processes and speciation in human culture occur in geographical space, as they also do in nature. In addition, they also occur based on collective memory and institutional support. Quite understandably, this sets the stage for a mosaic of cultural diversity. To better comprehend this mosaic, we must next understand the processes through which the growth rates of multiple quasispecies, or, if you wish, multiple axes of dissemination, stay in balance. 
Fig.2 Science and humanities dissemination axes: geographical profiles. Cultural life renews itself unevenly, resulting in regional varieties.

US news, three corpora, thousand dissemination axes, interactive visual online.
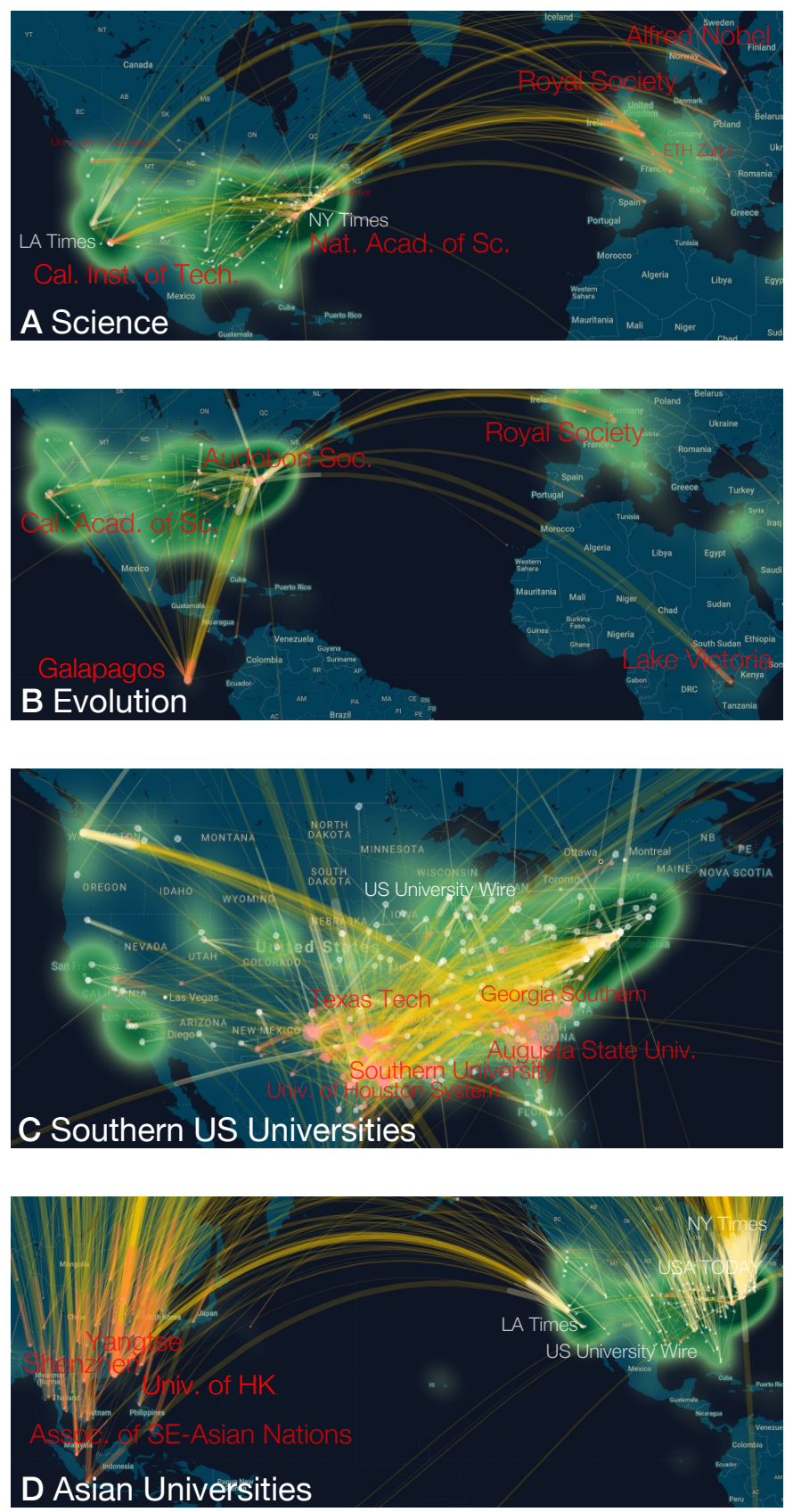

A The largest science dissemination axis in our corpora is themed on recognition and dominated by major research institutions along with the National Academy of Science and the Nobel prize that bridge interest in the various scientific disciplines. (S1.11) B The Galapagos, Lake Victoria, their birds, the California Academy of Science, and the Audubon and Royal societies dominate the discourse on evolution in public media giving its distinct geographical profile. (S1.129) C Regionally focused, but polycentric, this network unites many institutions in fixed geographical space. (S1.80) D China and south-east asia have many distinct dissemination axes in our corpora, among which this may just be the largest. It is focused on universities and history. (S1.42)

White: publishers

Pink: news content.

Lines connect publishers to content they published.

Green: dense zones in the overall corpus distributions. 
Fig.3 Science and humanities dissemination axes: institional and historical profiles. With their mission statements and the like, institutions such as publishers and funding agencies create their own variation-selection processes. Variation-selection may also emerge in the realm of collective memory.

US news, three corpora, thousand dissemination axes, interactive visual online.

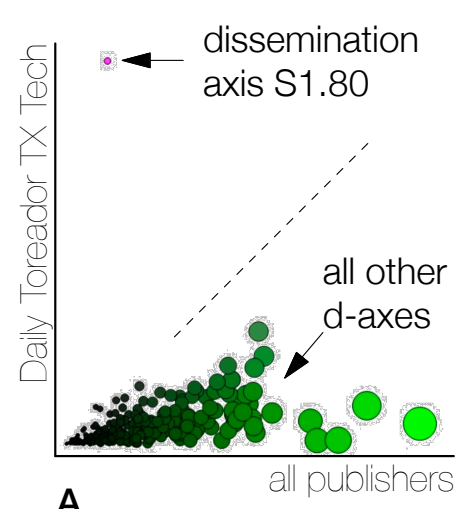

A

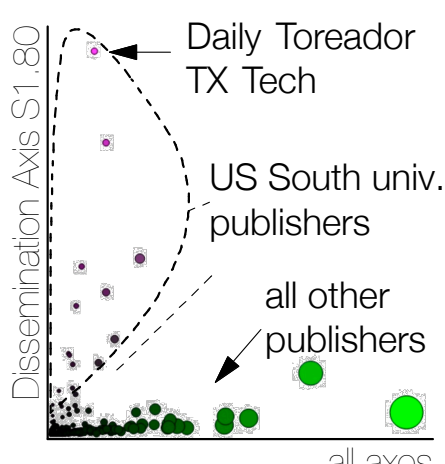

B

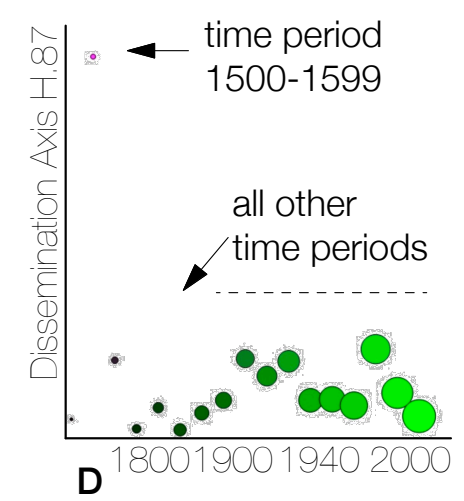

A,B Southern US

universities (Same

dissemination axis as in

Fig.1C) This geographically

bound network of publishers

creates its own

dissemination axis that they

specialize in (A), and that is

uncommon among other

publishers (B). (S1.80)

C,D 16th century

dissemination axes. These

dissemination axes taken

from our humanities corpus

root the public discourse in

the early modern epoch. The

most specialized among

them is the one on

Shakespeare and early

modern drama (A). Other

time periods are almost

absent from the

Shakespeare axis giving it a

distinct historical profile (B).

$(H .87)$

\section{Homeostatic Regulation}

The previous sections laid out the basics of variation-selection processes as applied to cultural life. The main insight is that ideas grow in groups of variants: Each group is a unit of evolutionary selection; each group is a quasispecies. We found that multiple groups coexist, and we interpreted this as a hint that some sort of self-regulation occurs. The growth rates of all groups must be kept in balance.

Given that we study human culture, let us tie this self-regulation back to homeostatic processes that occur in human perception. This setup immediately allows us to subdivide homeostasis into three sub-processes well known in neuroscience: habituation, discrimination, and sensitization. (These three time-dependent processes tell us that: first, people stop listening to repeated messages; however, second, they can learn to distinguish between similar messages; and third, they can regain interest if, based on some new experience, the message seems promising again.)

This theoretical setup leads us to a system of equations that, once applied to human culture, explains not only the coexistence of multiple quasispecies, it also explains waves of 
fashion, formative periods at the beginning of trends, diversity threshold situations, cycles of growth and reform, and the evolution, under certain circumstances, of a common ethos that unites entire bodies of knowledge.

The new equations again have equivalent formulations in the life sciences and chemistry. There, they are best known as Lotka-Volterra equations, named after Alfred Lotka and after the mathematician Vito Volterra, a pioneer of mathematical ecology (31).

The particular formulation of Lotka-Volterra equations that our reception theory leads to, and that, in absence of another name, could be called reception equations, do not stem from Lotka's own hand, but were put to paper at Oxford decades later where they helped develop what is today an entire field of study known as virus dynamics (32-34). Some people believe that ideas go viral. Here is a real mathematical equivalence derived from theory and supported by empirical testing.

\subsection{Waves of fashion are cycles of media activity and audience habituation}

Let us begin with the simplest case and add more complexity later. The first step is to model the effects of dissemination and habituation alone. This setup leads to a standard pair of Lotka-Volterra equations, familiar to most scientists. Volterra's work turned the equations into a textbook-case of life science. Students use the equations early-on in their education to explain the wavelike wobbling that one can observe in the sizes of prey and predator populations that consecutively outbalance each other. In our case, the equations are used to predict waves of fashion. Any quasispecies of ideas can serve as prey species; habituation is the predator (Fig. $4 \mathrm{~A}$ $B$ and Supplement). The interplay between ideas and habituation gives rise to waves of fashion.

Habituation not only leads to fashions, but also to diversification. This phenomenon is well known in biology. Thomas Insel showed that a tiny mutation in the chemistry responsible for habituation leads to divergent patterns of behavior and to simple but beautiful adaptations within the animal habitat (35). Insel studied prairie and montane voles. In one species, habituation to the animal's sexual mate was faster, and this allowed for more genetic recombination. Hence, habituation supports diversification.

Insel's discoveries also found many practical applications beyond voles and lab mice. Much testing was performed on humans because the related neurochemistry is easily administered through the air. The next section will test whether habituation in human culture also leads to cultural diversification. To achieve this, we must introduce the concepts of discrimination and sensitization.

\subsection{From formation periods and rapid turnover to collective breakthrough}

Habituation is often found in interplay with discrimination and sensitization. If these three processes are integrated, the above-mentioned reception equations are obtained (Eqn. 1 in Supplement). These equations explain cultural diversification processes in unprecedented clarity.

Consider the event that a fashion becomes popular. In response, habituation is activated and turns people indifferent. This brings about the decline of this fashion. However, it is important to remember that people can discriminate. They only become indifferent to the fashion in question, not to life in general. Meanwhile, variation has created variants that escape habituation. New fashions begin to grow. Where, initially, there was one fashion, now, there are multiple of them. 
Fig.3 Large-scale reception. Three homeostatic processes (habituation, discrimination, and sensitization) explain waves of fashion, periods of formation, diversity thresholds, and the transition from history dominated great people to more nuanced cultural diversity and collective rise to fame.

A Media activity and habituation lead to waves of fashion as modeled with Lotka-Volterra equations. B Waves of fashion found in the Chicago school of architecture. Museum exhibitions ride the waves. (Chicago school corpus, 105K perodicals and books, y=100 references) $\mathrm{C}$ Habituation, discrimination and sensitization lead to a double-phased evolution with a diversity threshold between the two phases, individual spikes on the left and collective rise on the right. During fromation, ideas do not lie dormant, but diversity. This is a textbook case of evolutionary dynamics known to occur in virology. D The phenomena are also found in empirical data, for example in the Chicago school at large. The peak on the left is dominated by one school. The rise on the right is a consequence of collective breakthrough. (Data: google Books, y=1/10M words)

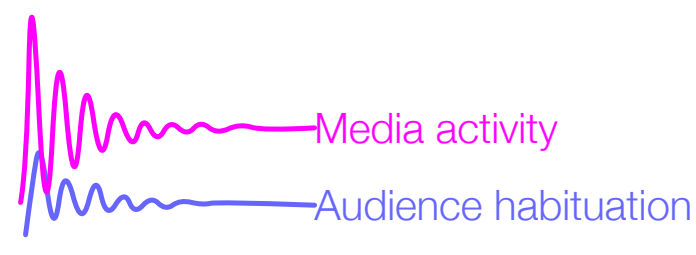

A Habituation cycles
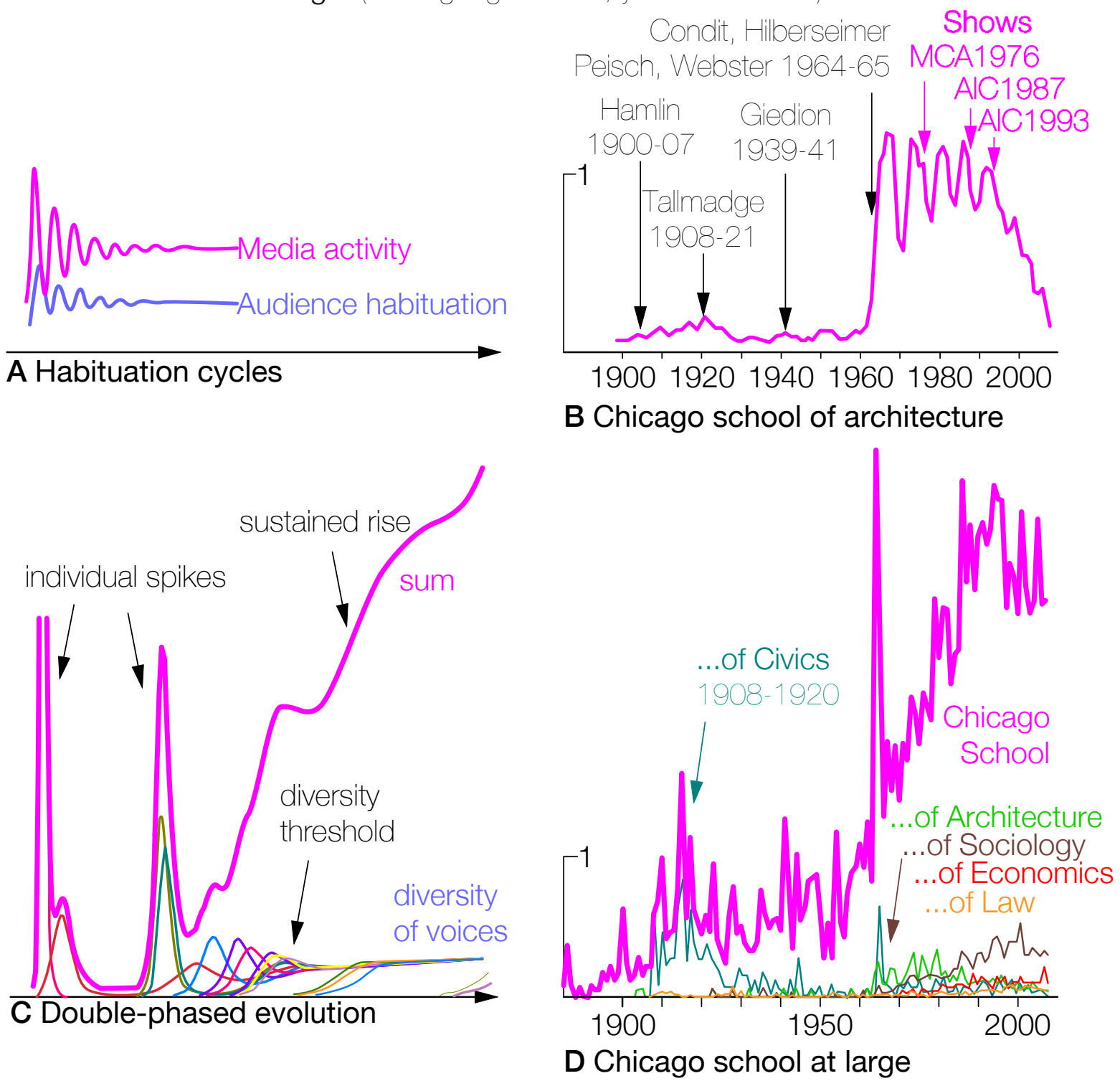
With multiple fashions coexisting, we must go on and consider the possibility that new fashions make people regain interest in old ones. This is a type of sensitization. Let us call it cross-sensitization. If such a process takes place, one can use the equations to predict that evolution has two distinct phases, formation and growth. During formation, habituation is active and leads to diversification. The phase of growth sets in when enough diversity is accumulated, such that sensitization disrupts habituation. However, because habituation is disrupted, diversification fails. The equations return to quasispecies behavior. The onset of each of these phases can be predicted from diversity threshold conditions.

These predictions can be tested against empirical data. Here again, the predicted phenomena are not uncommon. To begin with, formative periods are often followed by collective breakthrough. This phenomenon is frequently observed in human culture. Scholars of history already coined the term formative period. Now, this phenomenon can be explained as a result of processes that are everywhere at play in human and animal brains. In addition, the reception equations state that formative periods are periods of diversification, and they also state that the transition from formation to growth occurs after certain diversity threshold conditions are met. These predictions are tested in Figure 4 (Fig.4C-D and Supplement).

\subsection{Diversity and habituation regulate growth and recession cycles}

Every scientist should become acquainted with the phenomenon of diversification, crosssensitization, and growth. Let us consider a concrete example of immediate interest: There is physics, biology, geography, etc., but there also are natural sciences, physical science, human science, life sciences, climate science, etc. Science makes the link between disciplines explicit and interconnects audiences. The result is cross-sensitization by reference to a joint cause (Fig. $5 D$ below). The concept of cross-sensitization is easy to understand: Interest in life science could also spark interest in human science.

With respect to the term science it is possible to empirically show that there is more than formation and growth in one single cycle. In science, there are three consecutive cycles of growth and reform. They stretch over three centuries. During this evolution growth leads diversity loss, while stagnation allows diversity to rebound and to catalyze new growth (Fig.5 and Supplement). As above, the evolutionary model is again identical to the one for virus dynamics (32-34, Equation 1 in Supplement).

In absence of another name, I called this type cooperation diversity selection. The five main types of cooperation, (direct and indirect reciprocity, and spatial, group, and kin selection, 36), are thus now complemented by a sixth type of cooperation that is more than a special case of virus dynamics. Even more prominently, the same process is found in human culture.

Diversity selection can occur if the benefit of diversification is larger than its cost. However, are there any benefits that can consistently be gained from diversification? Yes. Diversification helps evolution search in multiple directions. Two hands can do more than one hand with ten fingers. Through diversification, evolution can grab with multiple hands, or it can, at once, take both the road and the road not taken. 
Fig.5 Growth and stagnation as diversification cycles. Diversification leads to growth, but growth reduces diversity and comes to a halt. Diversification restarts. These predictions are confirmed by measuring growth and Simpson's diversity index. In both simulation and empirical data, growth and diversity index move against each other. Diversity index stays between thresholds over three centuries of "science."

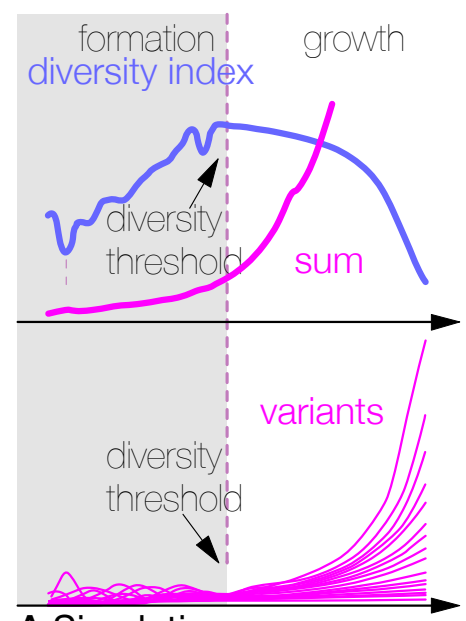

A Simulation.
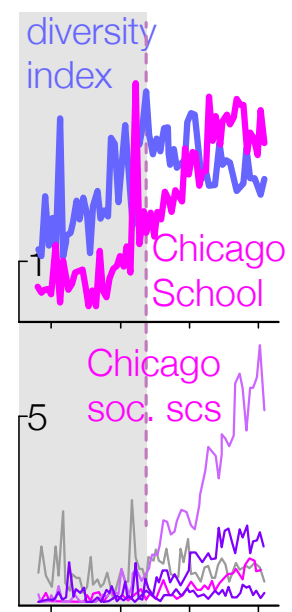

20001900

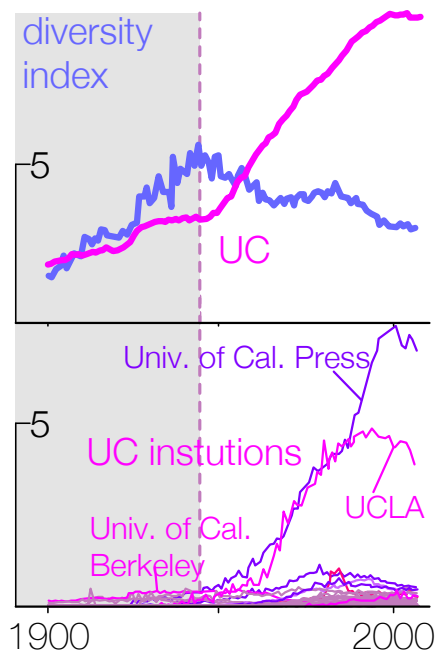

B Chicago, 60 y. C Univ. of Calif., 100 years

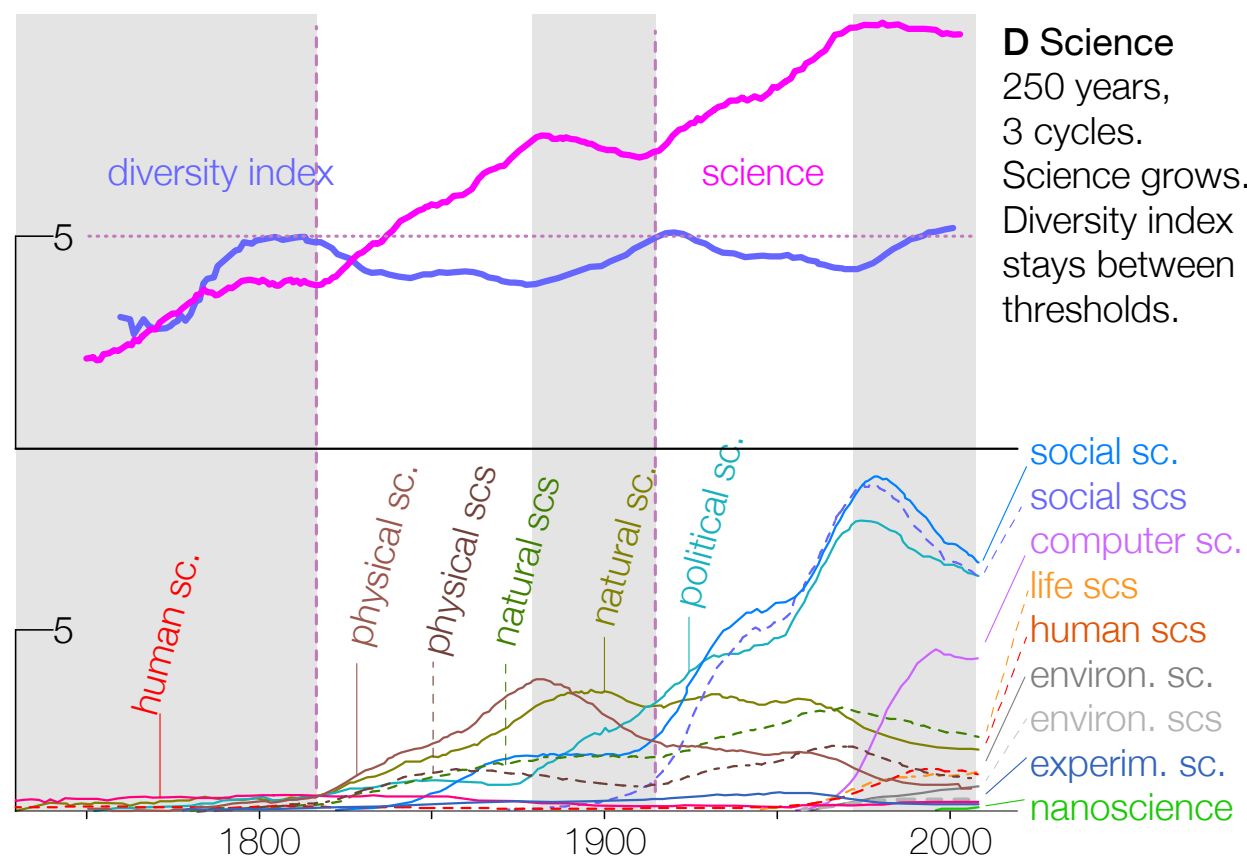

A Diversity and growth delimit each other. Simulation by Nowak et al. 1991, initially developed for a different application. B Diversification in Chicago schools of social science. "Chicago School"/"Chicago" y=1/1 M words, Chicago schools of social science $y=1 / 1 \mathrm{M}$ words, Diversity Index $y=1 / \mathrm{D}$. C Diversification in institutions of the University of California. "University of California"/"California" $y=2 / 100$ words; Diversity index $y=1 / D$; institution names $y=1 / 1 \mathrm{M}$ words. D Science and science branches. "Science" $y=2 / 10 \mathrm{~K}$ words; Diversity index y=1/D; Science branches y=1/1 M words. Data: google Books. 


\subsection{From creative origins to common ethos}

The reception equations can be further developed to take into account the distinction between dissemination drivers and those colorful attributes, the main role of which is to make the initial ideas appear more novel and diverse. There is a similar distinction in genetics. Cancer driver genes drive the growth of tumor and metastases, while many other genes are passed along and mutate, but only indirectly affect the disease.

New influential work shows that driver genes vary in the original tumor more than in metastases. This insight was recently backed with equations (37); and we also make this type of observation in human culture. There are trendsetters and followers. The first discover and rediscover, while the latter follow. A pair of examples is at hand: When evolutionism gained recognition, Wallace was already moving towards something new; and the type of humanistic endeavor that was called science in early modern English during the scientific revolution is no longer recognized as science. However, this is only anecdotal evidence. In my doctoral thesis, I have confirmed this phenomenon on a large scale for the Chicago school (13).

\section{Discussion}

One isolated transmission may add noise to a message, and one creative brushstroke may diminish an already finished artwork; however, when entire cultural communities think together, they transform noise and individual creativity into collective strategy. This is negative entropy in cultural life. Individuality is on one side of the equations, collective action on the other, and between them are dynamic balances and diversity.

Understanding culture as interplay between individuality and collective action has led us to large-scale dissemination and reception as well as variation-selection processes and cultural homeostasis, in which entire communities of people habituate to information, but also learn to discriminate, and they can be sensitized and cross-sensitized to certain stimuli.

The physical effects of these processes are many. Among them, we observed culture rise along dissemination axes with geographical, historical, and institutional profiles that, if plotted on logarithmic paper, translate into power laws. We saw that formative periods are phases of diversification and rapid turnover, followed by collective breakthroughs after diversity threshold conditions are met. Homeostatic processes over the course of those phases may lead to waves of fashion and to diversity selection as a sixth type of cooperation that accommodates cycles of growth and reform while a common ethos initiated by trendsetters is perpetuated across entire cultural communities. A single logical step sets off an avalanche of consequences.

The present article has not only proposed a theory. The theoretical postulates have been tested using linguistic, institutional, historical, and geographical data. These data were collected from written and spoken word as recorded in news and books, as well as social and mass media. In addition, the same theory logically applies to any other type of culture, no matter whether it evolves on large marketplaces or whether it takes shape among professional groups, for example of financers or scholars. With access to live data, automatic tools, and theory as developed here, it is now possible to evaluate and understand cultural change in real time; and consistent diversity thresholds may occasionally allow for cautionary forecasts.

The varieties equations are not only about individualism and collectivism, but also about diversity. Collectivism and diversity have sometimes been held to mutually exclude each other because of an unsettling uncertainty relation. At different scales, data may invite divergent 
interpretations that cannot be pursued all at the same time (38). Edward Simpson, whose diversity measure is employed in Figure 5, is only one of many who formulated this principle: Small data may point one way, aggregated data, may point the other.

This unity-diversity uncertainty is deeply rooted in the human mind; it is the etymological source for something as vast as the uni-verse and as intellectually fulfilling as uni-versity (with presently renewed concerns about diversity and inclusion). The same uncertainty may have left behind divergent interpretations for the Tower of Babel. Was the tower a symbol of growth and greatness, or did its builders begin to babble, loosing their common basis of communication?

The modern world may still face this problem of uncertainty. Globalization unites an increasing number of people while the seemingly opposite process, diversification, advances among them: serial mass production has become individualized, and mass media have given way to social media. The first process has inspired global economical institutions, the second postmodernism with its multitude of incompatible realities.

However, what is the precise relationship between the two processes? The varieties equations substantiate that diversification is the very source of sustainable collective growth. Diverse sociocultural groups are not alone; they need each other in order to grow. Diversity is most meaningful in the presence of inclusion.

After so many considerations, we must admit that our inquiries suggest that humans are not alone in yet another sense. Nature and culture are very much alike. Variation-selection processes and homeostasis unite physics, nature, and culture.

This also means that humanities research does not need to be separated from science, which has become silent reality over the last centuries. Science refers to any empirical or theoretical system of knowledge. On the other hand, humanities refer to the study of human culture. Science is a method, the scientific method. Humanities are a field of study. Method and field of study can well work together. The study of human culture, as practiced in this present article, is theoretical and empirical; it is a type of science.

You may say that there is also other humanities scholarship, and you are right. Therefore, while observing how categories of STEM and GLAM evolve through variation-selection processes and homeostasis, we must recognize that the study of life unifies research under a broader scientific umbrella. Researchers and scholars are diverse, but not alone. They jointly expand our understanding of life in many directions both in nature and culture.

\section{REFERENCES AND NOTES}

1. N.L.Komarova, M.A.Nowak, Language Dynamics in Finite Populations, Journal of Theoretical Biology 221, 445-457 (2003).

2. N.L.Komarova, M.A.Nowak, The Evolution of Grammar Acquisition, Journal of Theoretical Biology 209, 43-59 (2001).

3. M.A.Nowak, The Basic Reproductive Ratio of a Word, the Maximum Size of a Lexicon, Journal of Theoretical Biology 204, 179-189 (2000).

4. M.A.Nowak, J.B.Plotkin, D.C.Krakauer, The Evolutionary Language Game, Journal of Theoretical Biology 200, 147-162 (1999).

5. E.Schrödinger, What is Life? (Cambridge University, Cambridge, 2012).

6. A.J.Lotka, The law of evolution as maximal principle, Human Biology 17, 167-194 (1945).

7. A.Bejan, The Physics of Life (St.Martin's, New York, NY, 2016). 
DC Baciu, Cultural Life, OSF Preprints (May, 2020). DOI: 10.31219/osf.io/ad9fu

8. A.Bejan, Hierarchy in Nature, Society and Science (Springer, Basel, 2020).

9. Students are named in Acknowledgements.

10. C.Shannon, A mathematical theory of communication, Bell System Technical Journal 27, 379-423 (1948).

11. M.Eigen, Self-organization of matter and the evolution of biological macromolecules, Naturwissenschaften 58, 465-523 (1971).

12. A.Besnard, J.Caboche, S.Laroche, Reconsolidation of memory, Progress in Neurobiology 99, 61-80, (2012).

13. J.Henrich, The Secret of Our Success (Princeton, Princeton, NY, 2016).

14. D.C.Baciu, Chicago Schools: Authors, Audiences, and History, Prometheus 2, 2019.

15. A.R.Wallace, On the tendency of varieties to depart indefinitely from the original type, Linnean Society Zoological Journal 3, 53-62 (1858).

16. A.R.Wallace, The Malay Archipelago (Macmillan, London, 1969).

17. C.Darwin, On the perpetuation of varieties and species by natural means of selection, Linnean Society Zoological Journal 3, 46-53 (1858).

18. A.R.Wallace. On the natural history of the Aru Islands, Annals of Natural History 20, 473-477 (1857).

19. C.Darwin 1859. On the Origin of Species by Means of Natural Selection (Murray, London, 1859).

20. Aristotle, 5. Definitions, in Topica (University of Adelaide, Adelaide, 2015).

21. E.Domingo, P.Schuster, Quasispecies (Springer, Basel, 2016), pp 1-22.

22. V.Pareto, Cours d'Économie Politique (Rouge, Lausanne, 1896-7).

23. A.J.Lotka, The frequency distribution of scientific productivity, Journal of the Washington Academy of Sciences 16, 317-323 (1926).

24. G.K.Zipf, The Psycho-Biology of Language (MIT, Cambridge, MA, 1965).

25. S.T.Piantadosi, Zipf's word frequency law in natural language, Psychonomic Bulletin 21, 1112-30 (2014).

26. P.Giraud, The semic matrices of meaning, Social Science Information 7, 131-39 (1968).

27. T.K.Landauer, S.T.Dumais, A solution to Plato's problem, Psychological Review 104, 211-40 (1997).

28. I.Matveeva, G.Levow, A.Farahat, C.Royer, Term representation with generalized latent semantic analysis, Recent Advances in Natural Language Processing (2005).

29. J.A.Bullinaria, J. P. Levy, Extracting semantic representations from word co-occurrence statistics, Behavior Research Methods 44, 890-907 (2012).

30. R.H.MacArthur, E.O.Wilson, An equilibrium theory of insular zoogeography, Evolution 17, 373-387 (1963).

31. A.J.Lotka, Contribution to the theory of periodic reactions, Journal of Physical Chemistry 14, 271-74 (1910).

32. M.A.Nowak, R.M.May, R.M.Anderson, The evolutionary dynamics of HIV-1 quasispecies and the development of immunodeficiency disease, AIDS 4, 1095-1103 (1990).

33. M.A.Nowak, R.M.Anderson, A.R.McLean, T. F. W. Wolfs, J. Goudsmit, R. M. May, Antigenic Diversity Thresholds and the Development of AIDS, Science 254, 963-969 (1991).

34. M.A.Nowak, R.M.May. Virus Dynamics (Oxford University, Oxford, 2000).

35. T.R.Insel, L.E.Shapiro, Oxytocin receptor distribution reflects social organization in monogamous and polygamous voles, Proceedings of the National Academy of Sciences 89, 5981-5985 (1992).

36. M.A.Nowak, Five rules for the evolution of cooperation, Science 314, 1560-1563 (2006).

37. J.G.Reiter, A.P.Makohon-Moore, J.M.Gerold, A.Heyde, M.A.Attiyeh, Z.A.Kohutek, C.J.Tohkeim, A.Brown, R.M.DeBlasio, J.Niyazov, A.Zucker, R.Karchin, K.W.Kinzler, C.A.lacobuzio-Donahue, B.Vogelstein, M.A.Nowak, Minimal functional driver gene heterogeneity among untreated metastases, Science 361, 1033-37 (2018).

38. D.Baciu, thesis, University of Medicine and Pharmacy, Cluj-Napoca, 1982.

39. F.Bertels, C.S.Gokhale, A.Traulsen. Discovering complete quasispecies in bacterial genomes, Genetics 206, 2149-2157 (2017). 


\section{ACKNOWLEDGEMENTS}

D.Baciu, I.Matveeva, B.Capitanu, E.Dickson, D.Roth, N.Khedekar, S.Pande, A.Paturi, K.Kilari, S.Jagirdar, K.Bhatiya, A.Sharma, S.S.Murthy, S.Guhathakurta, P.Castro, C.Kumar, M.Kadam, D.Ghiurco, S.Naik, M.Tunga, S.Kashyap, K.L.Rajakumar, B.Subramani, R.Sukhadia, H.K.Shivappa. Grants: HathiTrust Research Center, scientific support and data, recipient D.C.Baciu; Swiss National Science Foundation, grant nr. 174833, recipient D.C.B.; The Fulbright Program, recipient D.C.B.; Swiss-American Society, recipient D.C.B.; Illinois Institute of Technology, recipient D.C.B.; Mellon Foundation, recipient A.Y.Liu. Author: D.C.B., main author. Interactive visuals by Interpretation Laboratory: D.C.B., director of lab, research concept and supervision, design and coding; Sihwa Park, GeoD and 7D (design); Cindy Kang, GeoD (design); Yichen Li, GeoD (design), 7D (coding); Junqing Sun, wikification of text data; Akshay Sunku, Zhenghao Zhao, Sunit Pravin Kajarekar, semantic testing, 7D and GeoD (coding). Competing interests: No conflicts of interest. Data and materials availability: Data, code, and interfaces upon publication released under creative commons license. Corpora: HathiTrust Research Center (Chicago school corpus); University of California Santa Barbara, Department of English, WE1S Project (science and humanities corpora); Google Books (Google Books data).

\section{Key publications}

Dan Costa Baciu. "Chicago Schools: Authors, Audiences, and History." Prometheus 2 (2019), p. 20-43. [Introduces the idea of entropic processes in human culture. Makes the connection between Shannon's definition of communication and negative entropy via variation-selection processes. Gives mathematics and testing with empirical data on the Chicago school.]

Dan Costa Baciu. "From Everything Called Chicago School towards the Theory of Varieties." Doctoral dissertation, Illinois Institute of Technology, April 2018. [Develops the theory of varieties as a theory of dissemination and reception with quasispecies and Lotka-Volterra equations, gives testing results, explains methods.]

Dan Costa Baciu. "The Chicago School: Evolving Systems of Value." Report for the Advanced Collaborative Support Project 06.2016-03.2017 at the Hathi Trust Research Center, October 2017. [Develops the theory of dissemination with the quasispecies equation; tests the theoy; and explains some methods and applications.]

Dan Costa Baciu. "Sigfried Giedion: Historiography and History of Reception on a Global Stage.” Ar(t)chitecture, Haifa: Technion Israel Institute of Technology 2016, p. 40-52. [Shows an application of the theory.]

\section{A note on this preprint}

Given that I propose a theory of dissemination, I take it as a duty to also popularize my work, disseminating my ideas in magazines and talking about my theory and empirical testing at many academic events. Only in the last five years, I presented at more than twenty occasions at conferences and in departmental seminar series. In addition, I published testing results, and I offered support to other researchers who wished to use my methods. Furthermore, editors of many journals have read my work, some of which has been blocked for months and years of peer review on end. This latter phenomenon is the reason why I decided to make this article available as preprint. 
Under these circumstances, the theory proposed in this article may also apply to the theory itself. Maybe you will soon find other versions and variants of my theory. If you stumble on something like that, ask yourself three questions: 1) Did the authors make the connection between Shannon communication, Aristotelian systematics, negative entropy, variation-selection processes, and homeostatic regulation? Is there one of the pieces missing? 2) Was the theory formulated in words as well as testable mathematics? 3) Do the authors empirically test any predictions?

Below, you can find an itemized list of the components that make my theory:

1) Variation-selection and quasispecies equation as a theory of dissemination (essential)

2) Homeostatic regulation and Lotka-Volterra type equations as a theory of reception (essential)

3) Empirical testing (essential)

One quasispecies of ideas

Multiple quasispecies of ideas

Polarization; clustering patterns are self-perpetuating

Geographical analysis of clusters

Categorical analysis of clusters

Fashion waves

Formation periods as periods of diversification

Diversity threshold conditions

Cross-sensitization

Diversification cycles

Common ethos

4) Link between Shannon's definition of communication, entropic processes, negative entropy, and variation-selection processes (result)

5) Link between variation-selection processes and Pareto distribution (result)

6) Link between variation-selection processes and Latent Semantic Analysis or other similar dimensionality reductions (result)

7) Mathematical equivalence between nature and culture when it comes to variation-selection processes, homeostasis, and diversification (result)

Evolution in nature; Wallace, Darwin, and island biogeography (historical context)

Aristotelian systematics; Genus and Differentia (historical context)

Quasispecies equation in genetics (historical context)

Virus dynamics equations (historical context)

Cancer gene evolution (historical context)

8) Diversity selection (result) 